\title{
A review of shallow seismic methods
}

\author{
Don W. Steeples \\ Department of Geology, University of Kansas, Lawrence, Kansas, U.S.A.
}

\begin{abstract}
Shallow seismic methods have historical roots dating to the 1930 s, when limited shallow refraction work was performed using the Intercept-Time (IT) method. Because of high costs and the general lack of appropriate equipment - particularly data-processing equipment and software - the shallow-reflection and surface-wave techniques did not catch on as quickly as the refraction techniques. However, since 1980 substantial progress has been made in the development of all of the shallow seismic approaches. The seismic-reflection method has been used increasingly in applications at depths of less than $30 \mathrm{~m}$, incorporating both the standard Common-Midpoint (CMP) method of the petroleum industry and the Common-Offset (CO) method, which was developed specifically as a low-cost technique for use in shallow surveying. In refraction studies, the Generalized Reciprocal Method (GRM) largely has replaced the classical intercept-time method, and tomographic approaches are rapidly gaining popularity. The Spectral Analysis of Surface Waves (SASW) has been developed by civil engineers, and surfacewave analysis involving many seismograph channels (MASW) recently has shown promise. With any of the shallow seismic methods, however, selecting the appropriate seismic recording equipment, energy sources, and data-acquisition parameters, along with processing and interpretation strategies, often is critical to the success of a project.
\end{abstract}

Key words shallow seismic reflection - Rayleigh refraction

\section{Introduction}

This paper describes some of the basic features of near-surface seismic methods applicable to shallow engineering, mining, and environmental projects. Such techniques are rapidly finding new applications in characterizing geologic, hydrologic, and stratigraphic conditions within 1 to $100 \mathrm{~m}$ of the Earth's surface. Prospecting for progressively smaller and shallower geologic targets is becoming possible as developments in research and instrumentation allow higher seismic frequencies to be recorded, engineering seismographs offer more channels, and data processing becomes less expensive.

Mailing address: Dr. Don W. Steeples, Department of Geology, University of Kansas, Lawrence, Kansas 66045. U.S.A.; e-mail: Dsteeples@ku.edu
The discussion presented here is not intended to be a thorough theoretical explanation or mathematical presentation of exploration seismic methods, which are readily available in many textbooks. The reader should know, however, that certain similarities exist among the various seismic methods and should be aware of the general limitations of the methods as well.

In the world of shallow geophysics, the seismic-reflection, seismic-refraction, and GroundPenetrating Radar (GPR) techniques are similar in some respects. Similarities to Crosshole Seismic Tomography (CST) and Vertical Seismic Profiling (VSP) exist as well. The likeness to electrical and potential-fields methods, however, is less substantial.

All seismic methods require sources to produce seismic energy and receivers (geophones, in this paper) to detect the energy after it travels through a volume of the Earth. Seismic methods are most sensitive to the mechanical properties of earth materials, but they are relatively insensitive to the chemical makeup of earth materials 
and the fluids they contain. In contrast, electrical and GPR methods are sensitive to contained fluids and to the presence of magnetic or electrically conductive materials. The measurable physical parameters upon which seismic methods depend are quite different from those important in the GPR, electrical, and magnetic methods.

Understandably, excavation is the oldest among the shallow-exploration techniques. Today, however, Baker et al. (1999a,b) have shown that seismic reflectors and refractors can be mapped, under some conditions, at depths of less than $1 \mathrm{~m}$. Likewise, high-frequency surface-wave methods can be used to examine the subsurface at depths of several meters (Xia et al., 1999a; Park et al., 1999a).

In general, the cost of excavating earth materials increases with depth. In contrast, the cost per unit area surveyed using near-surface geophysical exploration methods decreases with increasing depth. But the reduced financial expense is accompanied by a decrease in resolution capability with increased depth, which may be unacceptable to engineers seeking answers to their geologic questions. The decrease in cost is related to a decrease in resolution because the distance between observations in a well-designed survey is a linear function of resolution. Simply put, it doesn't make sense to position seismic lines $1 \mathrm{~m}$ apart when the lower limit of horizontal resolution is $10 \mathrm{~m}$. Horizontal detail requires closely spaced observations, which requires more work per unit area and therefore costs more per unit area. This is true for all of the geophysical methods.

Seismic methods date back to the early part of the 20th century. The seismic-reflection method in particular is a powerful geophysical exploration technique that has been in widespread use in the petroleum industry for more than 70 years. Since 1980, substantial progress has been made in all of the shallow-seismic methods. For example, the revolution in microelectronics has resulted in the construction of engineering seismographs and personal computers that can be used for data collection and processing and that permit the cost-effective use of seismic methods in a wide variety of near-surface applications. State-of-the-art seismic methods are quite advanced; however, they are underuti- lized in site characterization and engineering studies.

The near-surface seismic methods require careful attention to detail to avoid possible pitfalls with regard to data collection, processing, and interpretation (Steeples and Miller, 1998). Understanding the resolution limits of the techniques and planning seismic surveys around geologic objectives and resolution limits is in part the key to achieving success and avoiding pitfalls. Ensuring that the methods are increasingly cost effective relative to test drilling and/ or other geophysical methods also requires deliberate planning. Selecting appropriate seismic recording equipment, energy sources, and dataacquisition parameters often is critical to the success of shallow seismic projects, as is the selection of processing and interpretation procedures.

The principles that pertain to sound waves also apply to $P$-waves, which are compressional. Indeed, $P$-wave reflections can be thought of as sound-wave echoes emanating from underground. Elastic-wave energy can travel through materials in a variety of ways, e.g., as compressional $(P)$ waves, shear $(S)$ waves, or surface waves. These wave modes are based on wave theory and are distinguished by their particle displacements, transmission paths, and speeds of propagation. The $P$-waves propagating through the Earth behave in a manner similar to that of sound waves propagating in the air. When a $P$-wave comes into contact with an acoustical contrast, either in the air or underground, echoes (reflections) are generated. In the underground environment, however, the situation is more complex. Beneath the ground, some of the energy incident upon a solid acoustical interface is transmitted across the interface, some is refracted, and some is converted into shear waves. In addition to $P$ - and $S$-waves, two types of surface waves - Rayleigh and Love waves - may appear on seismograms. Rayleigh waves appear most often on $P$-wave surveys and are commonly called «ground roll» because of the relatively low-frequency rolling motion that they cause.

Seismic reflection and seismic refraction both have a place in shallow exploration. The physical theory upon which both methods are based is 
identical to that used in petroleum exploration, except that the spatial dimensions are scaled down. Additionally, bandwidth is scaled up to carry more information per unit of time so that the desired resolution can be obtained. Both seismic techniques allow the calculation of seismic velocity (although in different directions), provide a measure of geologic continuity, and usually permit an estimate of depth to the layers of interest.

Seismic methods employing $P$-waves are sensitive to the presence or absence of liquids to the extent that liquids affect mass density and seismic velocity. $S$-waves, on the other hand, are relatively insensitive to the presence of liquids. However, all seismic techniques are relatively insensitive to the chemical content of liquids. Rare exceptions may occur when a distinct interface is present between liquids that have substantially different acoustical properties, in a medium whose porosity is $25 \%$ or more.

Paradoxically, seismic-reflection methods and Ground-Penetrating Radar (GPR) are similar in concept but almost mutually exclusive in terms of the types of sites at which they work well. Both methods rely on the reflection of energy from underground features. Although GPR works well in the absence of electrically conducting materials near the Earth's surface, it is unable to penetrate materials that conduct electrical energy well. Seismic reflection, on the other hand, works best where the water table is located near the surface. For example, seismicreflection energy easily penetrates damp clays, which are excellent electrical conductors. But dry sands, which will not transmit high-frequency seismic waves easily, are penetrated readily by GPR.

\subsection{Seismic refraction}

The seismic-refraction method requires that the survey area contain earth materials that increase in seismic velocity as depth increases. The analysis of refraction data becomes more complicated when the materials contain layers that dip or are discontinuous. For shallow applications in which low-velocity layers are encountered within a few meters or tens of meters of the Earth's surface, the increasing-velocity requirement is a severe constraint. For example, a sand layer that lies under a clay layer in an alluvial valley commonly has a lower seismic velocity than does the clay layer; therefore, seismic refraction cannot be used without producing erroneous results. In addition to the velocity-inversion problem, a blind zone (i.e., where a layer is too thin to appear as a first arrival on a seismogram) can result in erroneous results. Other problems not treated well by seismic refraction include lateral velocity changes over small distances and the abrupt termination of geologic beds.

The field methods used in refraction studies include reversed refraction, single-ended profiles that do not allow the analysis of dip, and classic fan-shooting of the type used in the oil industry for salt-dome exploration. For some near-surface applications such as finding the edges of buried garbage dumps and locating near-surface voids, the possibility of three-dimensional fan shooting should be considered. The refraction technique is usually less expensive than the seismic reflection method. However, in areas in which both techniques work well, reflection can be expected to provide more detailed results.

The main variations among refraction methods lie in the user's approach to interpretation; hence, in data processing. The classical refraction method is the intercept-time method described in virtually all exploration geophysics textbooks. Other interpretation methods include the reciprocal method (Hawkins, 1961), based on Edge and Laby (1931), and the delay-time method (Nettleton, 1940). Palmer (1980, 1981) developed the generalized reciprocal method. An excellent reference by Lankston (1990) discusses modern refraction techniques, including the GRM.

\subsection{Shallow seismic reflection}

The success of seismic-reflection techniques depends on the existence of discrete velocity and/or density changes in the subsurface. This observation is identical to the perception that a sound wave in air does not echo unless it en- 
counters something solid. Discrete changes in seismic velocity or mass density are known as acoustical contrasts. The measure of acoustical contrast is formally known as acoustic impedance, which is the product of mass density and seismic velocity. In most cases, acoustical contrasts occur at the boundaries between geologic layers or formations, although manmade boundaries such as tunnels and mines also represent contrasts.

The classic use of seismic reflection involves identifying the boundaries of layered geologic units. Notably, the technique also can be used to search for anomalies such as isolated sand- or clay lenses and cavities. The technique is rapidly becoming more cost-effective, bringing new applications as resolution improves. Steeples and Miller (1990) provide an overview of seismic reflection as applied to shallow exploration problems.

The seismic reflection method, in use for over 70 years, is a powerful underground exploration technique (Telford et al., 1976; Coffeen, 1978; Sheriff, 1978; Waters, 1987). Until the 1980 s, however, it was not used to target areas shallower than $30 \mathrm{~m}$. The work of Jim Hunter and Susan Pullan and their colleagues at the Geological Survey of Canada (Hunter et al., 1984; Pullan and Hunter, 1985) and Klaus Helbig (Doornenbal and Helbig, 1983; Jongerius and Helbig, 1988) and his students at the University of Utrecht in The Netherlands was instrumental in developing shallow seismic reflection procedures.

The Common-Offset (CO) optimum-window method (Hunter et al., 1984) was used widely and routinely in engineering and groundwater applications in the 1980s and 1990s. However, Common-Midpoint (CMP) methods have eclipsed $\mathrm{CO}$ methods because of the availability of relatively cheap seismic processing software and the continuing decrease in computational costs. A data set collected with CMP processing in mind can be processed using $\mathrm{CO}$ methods by instructing the CMP processing software to sort the data into $\mathrm{CO}$ displays.

Moreover, shallow CMP and CO seismic reflection profiling are becoming less costly and are being adopted more often as personal computers are used increasingly to process CMP data efficiently (Somanas et al., 1987). Whereas the CMP method has signal-to-noise $(\mathrm{S} / \mathrm{N})$ ratio advantages in some areas, the computer-processing time required is often substantial. The $\mathrm{CO}$ method has the advantage of offering decreased processing costs and, sometimes, higher resolution, because CMP processing tends to decrease the bandwidth as a result of the «stretch" that occurs during the normal moveout phase of data processing (Miller, 1992).

\subsection{Seismic borehole tomography}

Seismic tomography employs the same mathematical inversion approach used by those in the medical profession who use $\mathrm{X}$-rays to image features inside the human body (e.g., computed axial tomography or CAT scan). The corresponding seismic technique involves timing large numbers of raypaths between boreholes, although it is common for surface-to-borehole and/or borehole-to-surface raypaths to be timed as well. The technique is computationally intensive as well as costly because boreholes are required. Nevertheless, it often produces a very detailed velocity model of the area between boreholes. Tomography has been used to study the interior of the Earth from scales of thousands of kilometers to tens of meters (Humphreys et al., 1984; Clayton and Stolt, 1981). Data can be augmented by measuring rays that reach various places on the Earth's surface from a number of depths within the borehole(s).

The principal advantage of seismic tomography over surface seismic techniques is improved resolution. Increased resolution comes in part from the broader bandwidths of the recorded energy, which is made possible by the excellent coupling of the seismic source and receivers to the competent earth materials in the borehole. The remaining improvement comes from the lower attenuation of seismic waves as they traverse earth materials at greater depths.

\subsection{Vertical Seismic Profiling (VSP)}

Vertical seismic profiling is seldom used alone; rather, it is used along with other tech- 
niques to improve the interpretation of seismic reflection data. Commonly, in VSP a string of hydrophones, three-component geophones, or three-component accelerometers is deployed inside a borehole. A surface-seismic source is located within a few seismic wavelengths of the borehole. The technique allows the accurate determination of the one-way traveltime of the seismic energy to various geologic units. It also allows the analysis of attenuation and acoustic impedance, both of which are needed to construct synthetic seismograms. Synthetic seismograms are used for comparison to actual seismic-reflection data to identify specific geologic formations and to refine depth estimates.

Transposed VSP involves the use of a source located within the borehole. Receivers, usually geophones, are placed at the Earth's surface. Transposed VSP has the advantage of being faster and cheaper than traditional VSP, provided a suitable seismic source can be used in the borehole repeatedly, at varying depths, without destroying the borehole.

In igneous and metamorphic terranes, a significant use of VSP is for the detection, location, and effective assessment of the permeability of productive fractures that intersect a borehole. Citations concerning VSP in the technical literature include Galperin (1974), Balch and Lee (1984), and Hardage (1983).

\subsection{Why are shallow seismic methods not used more widely?}

Several factors have prevented surface seismic methods from being the technique of choice for typical near-surface investigations. Until about 1990 , the techniques were prohibitively expensive, particularly seismic reflection. However, acquisition and recording systems offering an acceptable dynamic range and a broad bandwidth are now available to record the high frequencies necessary for shallow reflection work.

In shallow media, attenuation tends to be very high, particularly in arid regions in which the water table is deeper than $10 \mathrm{~m}$. In many areas, the acoustical impedance contrast is not strong enough at the geologic layer boundaries to produce recordable seismic reflections.
In addition, two-dimensional (2D) reflection surveys provide information only in the vicinity of the vertical plane that contains the seismic shot and the geophones that record the shot. Although three-dimensional (3D) shallow reflection surveys are now documented in the literature, they remain very expensive because areal arrays of closely spaced geophones on the Earth's surface are required (Büker et al., 1998).

The complex velocity structure of near-surface materials, along with their heterogeneous nature, tend to make the processing and interpretation of shallow seismic data difficult. This is compounded by the problem of ensuring that the geophones are coupled to the ground well enough so that the high frequencies can be recorded. Moreover, environmental restrictions on shallow seismic sources sometimes limit their ability to provide high-frequency broadband energy.

\section{Useful near-surface applications of seismology}

\subsection{Locating buried objects and voids}

Reports in the refereed literature are limited concerning the use of seismology to locate voids. Seismic research conducted to detect cavities resulting from salt-solution mining (Cook, 1965), lava-flow tunnels (Watkins et al., 1967), natural caverns (Rechtien and Stewart, 1975), and abandoned subsurface coal mines (Fisher, 1971; Hasbrouck and Padget, 1982) has met with limited success. Most of the research encompassing coalmine detection has involved refraction seismology or $S$-wave reflection seismology.

Most researchers using seismic techniques for cavity detection cite three phenomena as evidence of a cavity: 1) free oscillation or resonance of the cavity walls; 2) anomalous amplitude attenuation, and 3) arrival-time delay (Cook, 1965; Watkins et al., 1967; Godson and Watkins, 1968; Fisher, 1971). Biot (1952) found that the resonant frequency $(f)$ of a cylindrical borehole in an infinite solid is related to the diameter of the borehole $(D)$ and the shear-wave velocity $\left(V_{s}\right)$ of the medium by the relation 
$D=V / 1.55 f$. This relationship is true for a homogeneous medium such as a basalt layer, shale, or alluvium, but does not appear to be applicable to the coal-mine case because the cavity is bounded by three different materials (Fisher, 1971). The bottom of the cavity is composed of underclay, the sides of coal, and the roof of overlying strata, all of which possess different elastic properties.

Cook (1965) found that seismic energy transmitted through a cavity and reflected from a deeper horizon was attenuated more than energy reflected from the same horizon but not passing through a cavity, thus giving rise to a seismic amplitude «shadow». Robinson and Coruh (1988, p. 215) show an example of seismicreflection data in which a coal mine produces a low-amplitude shadow effect for reflections originating from layers deeper than the coal bed. Some success has been achieved in locating water-filled coal-mine cavities at depths of less than $15 \mathrm{~m}$ by using high-resolution $P$-wave reflection seismology techniques (Branham and Steeples, 1988; Miller and Steeples, 1991). The results reported by Branham and Steeples (1988) were based on the absence of a seismic reflection from a mined-out coal bed at a location where the coal bed produces a strong reflection. Anomalous seismic amplitudes also have been observed above lava tunnels (Watkins et al., 1967).

Because of the elastic properties of fluids, seismic shear waves theoretically will not propagate through voids or water-filled cavities and may therefore be used for cavity detection. Strong $S H$ waves have been generated and good reflections received from the tops of brine cavities $150 \mathrm{~m}$ (490 ft) deep (Cook, 1965). Shearwave reflections also have been used to evaluate the resources of a shallow coal seam (Hasbrouck and Padget, 1982).

Seismic refraction has been used to detect cavities, but with limited success (Fisher, 1971). A limited amount of work suggests that surface-wave phase anomalies might be used to detect near-surface voids (Steeples and Miller, 1988). Similar phenomena have been noted by Rechtien and Stewart (1975). Park et al. (1999c) employed surface waves to look for voids.

\subsection{Identifying fractures}

Fracture detection is among the classic uses of seismology. For example, fractures often contain preferred contaminant migration pathways. Engineering applications depend on the recording of higher frequencies and broader bandwidths than do classical seismic methods, however. The use of $P$-wave reflection techniques to detect shallow faults with offsets as small as 2 or $3 \mathrm{~m}$ is not exceptionally difficult. Likewise, the detection of shallow dissolution collapse features on the scale of a few meters horizontally and vertically is more or less routine. Recent work by Miller et al. (1999a) showed that shallow dissolution features could be detected by the Multichannel Analysis of Surface Waves (MASW) at a proposed power-plant site in Alabama in the southeastern U.S.A. Joint patterns and strike-slip faults could be detected using three-component seismic methods whose resolution is superior to that available to the technology now commonly in use.

\subsection{Mapping bedrock surfaces beneath landfills (i.e., garbage dumps)}

Landfill materials typically attenuate seismic waves much more quickly than do natural geologic materials. Therefore, using seismic methods is more difficult within a landfill than adjacent to one.

Nonetheless, several approaches can be taken. For example, the GRM could be used. Another method would be to undershoot the area by placing the seismic source on one side of the landfill and the geophones on the other. This normally involves wide-angle reflections whose phase distortions may be significant (Pullan and Hunter, 1985), thus making data processing more difficult. Additionally, the elastic discontinuities at edges of landfills are typically distinct enough to be detected by SASW or MASW techniques.

Refraction can also exploit the dry, loose nature of landfill materials. Mapping the lateral boundaries (edges) of individual cells, even when mapping the bottom topography of the same cell might prove very difficult, therefore becomes possible. As noted previously, refraction 
fan-shooting could be used to search for landfill boundaries.

A vertical or horizontal borehole can also be used to place sources or geophones on the edge of the landfill. Travel-time anomalies could then be used to analyze depth and/or velocity variations in the vicinity of the landfill.

\section{Shallow seismic fundamentals}

The basic instrument used in seismic studies is a seismograph, which is analogous to a stereo music system. The higher the quality of the music system, the better the listener is able to discriminate subtle musical nuances. Likewise, the better the dynamic range of a seismograph is, the easier it is to distinguish subtle geologic features. A stereo music system has controls that enhance high frequencies (treble) and/or low frequencies (bass). A seismologist selects the frequencies that are to be enhanced during processing, depending on the depth and size of the underground geologic features of interest and the frequencies generated by the source.

To detect small geologic features, a seismograph must be capable of recording and enhancing high-frequency sound waves in the presence of low-frequency noise. The use of high-frequency seismic waves (i.e., > $80 \mathrm{~Hz}$; Sheriff, 1991) in reflection seismology is known as «high-resolution» seismic exploration.

A single layer over an infinitely thick medium represents the simplest case of seismic reflection (fig. 1). A seismic-wave source imparts energy by explosion, mass drop, vibration, or projectile impact at or just below the Earth's surface. Waves then progress hemispherically away from the source point. Following Fermat's Principle of Least Time, a particular raypath originating at the source will pass energy through the subsurface layer, bounce off an acoustical contrast, and return an echo to the geophone at the surface. In fig. 1, the path of least time will include a reflecting point midway between the source and the geophone. The angle of incidence will be equal to the angle of reflection from the reflecting layer.

The shallow seismic reflection technique sometimes can detect several layers in the Earth

\section{Simple reflection from bedrock}

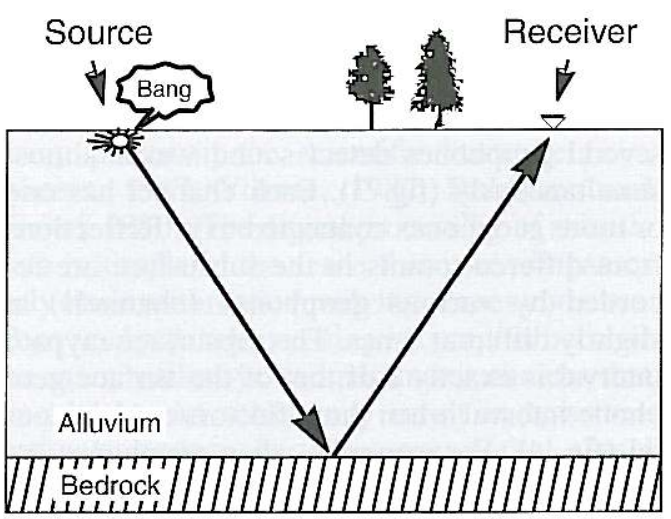

Fig. 1. The simplest case of seismic reflection. Layer 1 is an acoustical discontinuity.

\section{Two-layer reflection from bedrock}

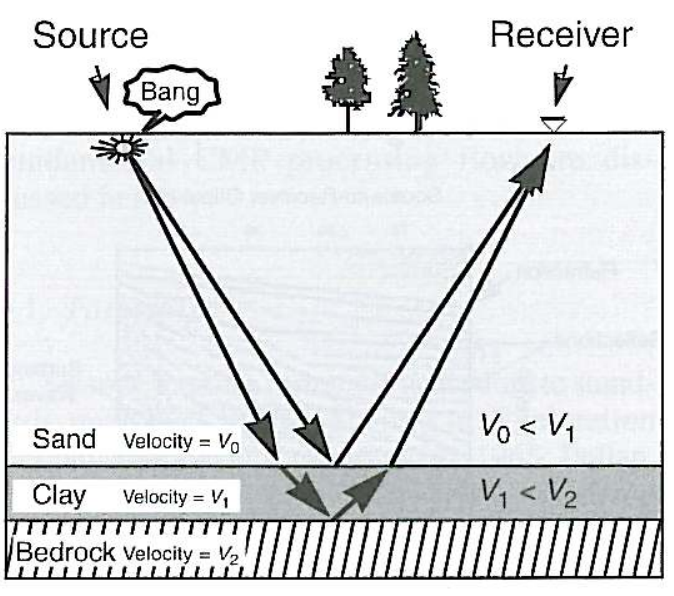

Fig. 2. Oversimplified figure depicting rays reflected from three layers. In general, raypaths are deflected from the straight lines at the boundaries between layers according to Snell's law.

(fig. 2). The raypaths are deflected at velocity discontinuities according to Snell's law. Several wave types and, at times, several layers contribute to the complexity of the seismograms. In 
most cases, refracted waves, surface waves known as «ground roll», and $P$-waves that are converted into $S$-waves at subsurface interfaces will also appear on seismograms.

In the case of the multichannel seismograph, several geophones detect sound waves almost simultaneously (fig. 3). Each channel has one or more geophones connected to it. Reflections from different points in the subsurface are recorded by various geophones (channels) at slightly different times. The subsurface raypath interval is exactly half that of the surface geophone interval when the reflectors are horizontal (fig. 4). For example, when geophones are spaced at 4-m intervals at the Earth's surface, the reflections will emanate from locations on the reflector, centered $2 \mathrm{~m}$ apart.

With the advent of digital recording in the 1960 s, the capability of adding traces together was realized (Mayne, 1962). From a geometric point of view, when sources and geophones are placed properly, some of the seismic traces will sample the same point in the subsurface (see

\section{Seismogram (example)}

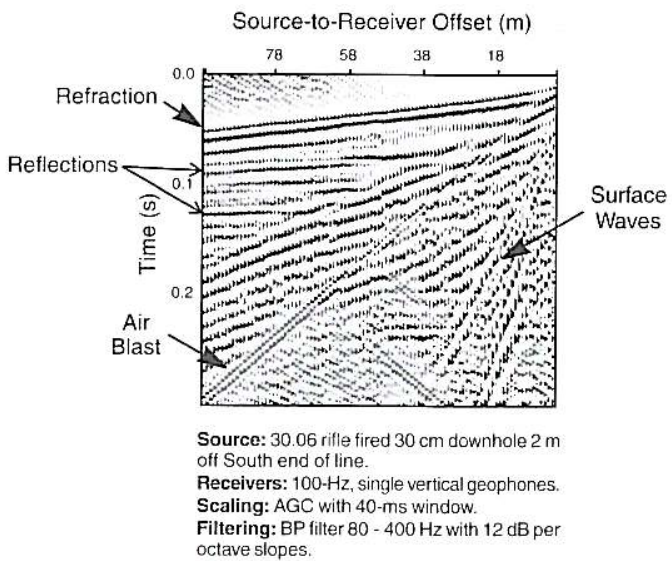

Fig. 3. Field seismogram (unprocessed) showing a bedrock reflection at about $53 \mathrm{~ms}$. The hyperbolic shape of the shaded zone is characteristic of simple reflections. The carlier-arriving energy is from air blast and from direct arrivals passing through near-surface alluvium. Geophone offsets are $3 \mathrm{~m}$ for the two traces on the inside and $16 \mathrm{~m}$ for the farthest traces.

\section{Simple reflection raypaths}

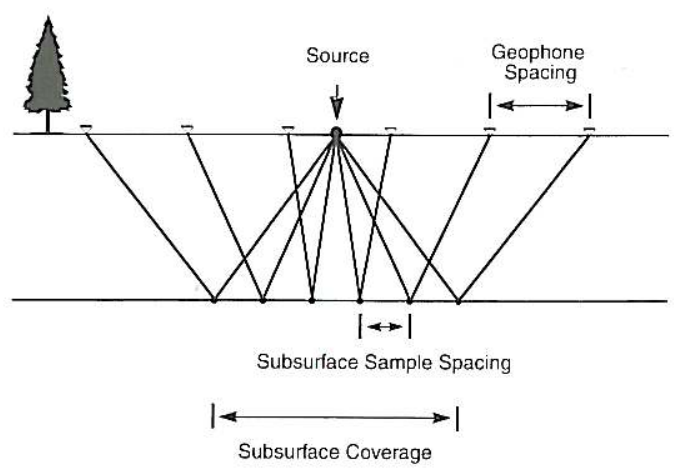

Fig. 4. Schematic of reflection raypaths in the case of a single layer. A six-channel seismograph was used. Note that the Common-Depth-Point (CDP) spacing is exactly one-half that of the geophone spacing.

\section{CMP concept}

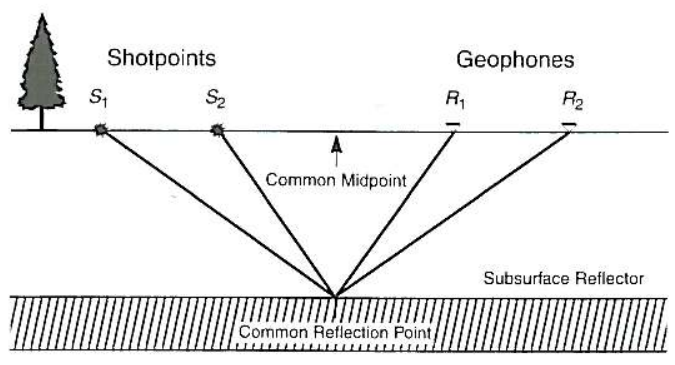

Fig. 5. Illustration of the Common-Depth-Point (CMP) concept. In the case of a 24-channel seismograph with shotpoints occurring at all geophone locations, the subsurface reflection points will be sampled 12 times, resulting in 12-fold CMP data after processing.

fig. 5). Such a point in the subsurface is properly called a Common-Reflection Point (CRP). The point on the Earth's surface midway between the source and the geophone is called the Common Midpoint (CMP). The CMP method is used extensively in the petroleum industry because of its capability to enhance data by adding («stacking») together traces that have a CRP. After correcting for the different path lengths and stacking the CRP traces together, the reflec- 
tion signal is enhanced by the square root of the number of traces stacked. The number of traces added together from a particular location is known as «CMP fold». This recording technique is sometimes called the Common-Depth Point (CDP) method, particularly in the older seismic-reflection literature.

The seismic-reflection method is used to calculate the spatial configuration and sometimes the lithology of geologic units. Figure 6 is a schematic example of a river valley with a bedrock surface containing a sand lens. The positive peaks of the seismic waves are blackened to assist with the geologic interpretation. The deeper the sand lens is buried below the surface, the more difficult it is to detect, because of the attenuation of the higher frequency components of the energy. However, the physical principles remain the same.

\section{Geologic model with seismic reflection data superimposed}

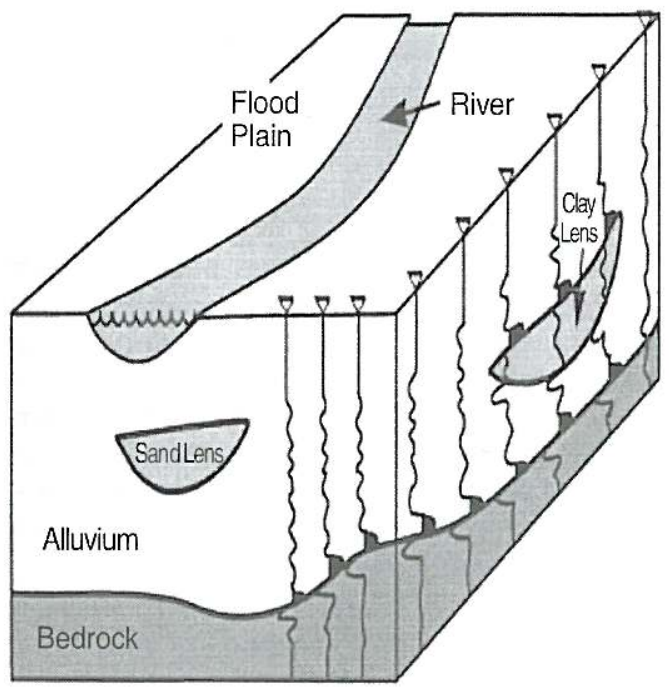

Fig. 6. Combining three-dimensional geology with a conceptual seismic section. The geology is interpreted from the coherent blackened peaks on the seismic section. Seismic data are processed to emulate the way in which they would appear when shotpoints and geophones are located at the same point on the Earth's surface.

\section{Processing shallow reflection data}

The goal of processing CMP seismic-reflection data is to enhance reflections and attenuate all other waveforms. Details are available in many places in the scientific literature (e.g., Waters, 1987; Yilmaz, 1987; Robinson and Treitel, 1980). The digital processing of seismic data is simple in the sense that it encompasses only basic arithmetic involving zeroes and ones. Conversely, data processing is complex because the many pathways and parameter choices that an analyst can select may result in less than optimal answers. In this paper, digital signal processing will be treated in ordinary language.

Seismic data processing can be broken into five basic categories of manipulation, including editing and sorting, static correction, geometrical correction, filtering, and enhancement by stacking. The categories of processes are illustrated in the following sections. Before a qualitative discussion of processing is launched, note that the output desired is an accurate acoustic image of the Earth's subsurface. A wide variety of filtering and display procedures can be employed to enhance the appearance of reflections. However, only those techniques essential to the fundamental CMP processing flow are discussed here.

\subsection{Formatting}

Seismic data are formatted according to standards published by the Society of Exploration Geophysicists (SEG) (Barry et al., 1965; Pullan, 1990). Often, data processing can be hindered by a user's lack of knowledge about the format in which data have been collected and stored. In fact, inadequate field notes and data-formatting problems are two of the principal barriers to effective data processing and archiving.

\subsection{Editing}

Engineering-seismograph systems contain recording channels ranging from 12 to several hundred. During the course of a seismic survey involving many shotpoints and recording chan- 
nels, some of the resulting seismic traces may not contain any data but may contain useless noise. Bad traces such as these can be caused by malfunctioning amplifiers, dead geophones, wires that have short-circuited or broken within cables, improperly planted geophones, or geographical factors such as the presence of rivers or paved roads.

Noisy traces can have a degrading effect upon processed data and should be removed by EDITing. This process involves turning the noisy trace off so that the data-processing package will not use it - or setting all of the data in a trace to zero. Sometimes only part of a trace is removed so that air blast, refractions, or ground roll can be removed. This procedure is known as a MUTE. Muting requires the tapering of the signal to zero at the edges of the muting window by means of a mathematical smoothing function. This is done so that discontinuities in the data, which may lead to processing artifacts during filtering operations, can be avoided.

\subsection{Sorting}

Engineering seismographs record the traces from a seismic shot into a specific block of Random-Access Memory (RAM). The traces then are transferred to a digital storage medium. To arrange the traces in some specified order according to a particular property such as common shot gathers, common midpoint gathers, or common offset gathers, the data are SORTed. When the editing and sorting have been completed, the processor may wish to display data from which bad traces have been removed, reversed polarities have been corrected, or muting has been performed.

\subsection{Statics}

Processes that involve a time shift for an entire seismic trace are termed static corrections. The datum static is an elevation correction used to compensate for the different topographic elevations of the shotpoints and geophones along the line. The second major source of variation requiring static correction arises from variations in near-surface seismic velocity from one point to another along the seismic line. Both types of static correction are made for seismic sources as well as geophone locations.

\subsection{Geometrical corrections}

Because CMP-sorted traces have different geophone offsets from the shotpoint, a correction known as Normal Moveout (NMO) is made to compensate for the variable subsurface path lengths of the seismic reflections at different geophone locations. Using NMO corrections properly requires the analyst to know the velocity of the seismic waves in the media above each reflector and to know with accuracy the locations of the shotpoints and the geophones. Consequently, velocity analysis is an important part of the NMO step during data processing. A proper NMO correction yields the two-way reflection time to a reflector, independently of the shotpoint-to-geophone offset. The NMO-corrected CMP traces then can be stacked to enhance the reflections.

\subsection{Filtering}

Data can be filtered either before or after CMP stacking. Filtering includes processes that discriminate against parts of the data based on parameters such as apparent velocity and frequency. Some filtering processes such as apparent velocity filters (i.e., frequency-wavenumber filtering) depend upon the properties of several adjacent traces, whereas frequency filtering depends upon only a single trace.

\subsection{Stacking}

Stacking is a process that adds traces together digitally. When the steps prior to stacking are completed properly, stacking enhances the reflections in such a way that S/N improves as the square root of the number of traces stacked together. 